Louisiana State University

LSU Digital Commons

Faculty Publications

Department of Biological Sciences

$1-5-2018$

\title{
NextGen VOICES: Research resolutions
}

\author{
Felicia Olmeta-Schult \\ Washington State University Vancouver \\ Lauren Massa Segal \\ University of Nebraska-Lincoln \\ Sam Tyner \\ lowa State University \\ Twila Alexandra Moon \\ University of Colorado Boulder \\ Ryan Dz Wei Chow \\ Yale School of Medicine
}

See next page for additional authors

Follow this and additional works at: https://digitalcommons.Isu.edu/biosci_pubs

\section{Recommended Citation}

Olmeta-Schult, F., Segal, L., Tyner, S., Moon, T., Chow, R., Chakrabarty, P., Pacesa, M., Podgornaia, A., Chen, J., Singh, B., Cao, B., Sidhu, R., Tan, B., Sood, P., Parker, S., Scult, M., Van Haute, D., Konstantinides, N., Schwendimann, B., Srivastava, S., Fiorenza, R., Dutton-Regester, K., Hale, R., Polat, E., Lau, E., Mayer, A., \& White, E. (2018). NextGen VOICES: Research resolutions. Science (New York, N. Y.), 359 (6371), 26-28. https://doi.org/10.1126/science.aar7504

This Letter to the Editor is brought to you for free and open access by the Department of Biological Sciences at LSU Digital Commons. It has been accepted for inclusion in Faculty Publications by an authorized administrator of LSU Digital Commons. For more information, please contact ir@lsu.edu. 


\section{Authors}

Felicia Olmeta-Schult, Lauren Massa Segal, Sam Tyner, Twila Alexandra Moon, Ryan Dz Wei Chow, Prosanta Chakrabarty, Martin Pacesa, Anna Igorevna Podgornaia, Jennifer Chen, Bipin Singh, Bo Cao, Rishi Raj Singh Sidhu, Bryce W.Q. Tan, Prashant Sood, Stuart Parker, Matthew A. Scult, Desiree Van Haute, Nikos Konstantinides, Beat A. Schwendimann, Sudhakar Srivastava, Raffaele Fiorenza, Ken DuttonRegester, Rachel Hale, Emre Ozan Polat, Edward Lau, Audrey L. Mayer, and Easton R. White 
A natural historian resolves to better explain the value of museum collections to the public.

engagement in politics must expand beyond matters pertaining to the NIH budget. We can begin by pressuring our representatives and making our voices heard. But to initiate the path toward systemic change, we must ultimately cultivate challengers of the political status quo from within our research communities. It is time to bring our passion from the lab bench to the podium.

\section{Ryan Dz-Wei Chow}

Yale School of Medicine, New Haven, CT 06510, USA Email: ryan.chow@yale.edu

My New Year's resolution for biologists: Do a better job explaining to the public why natural history museum collections are important. Natural historians are sometimes misunderstood as hunters or stamp collectors, but their collections, when curated ethically and legally, can open up a world of knowledge about our planet.

\section{Prosanta Chakrabarty}

Museum of Natural Science, Louisiana State University, Baton Rouge, LA 70803, USA.

Email: prosanta@Isu.edu

Deep sequencing and genome-editing technologies make personalized medicine, gene therapies, and transgenic livestock possible. Yet manipulation of genetic material carries a social stigma, due to ethical and ecological concerns. To overcome this stigma, the gene-editing community needs to better communicate the implications (good or bad) of our research to the public.

\section{Martin Pacesa}

Department of Biochemistry, University of Zurich, 8057 Zurich, Switzerland.

Email:m.pacesa@bioc.uzh.ch

\section{Interdisciplinary collaboration}

In 2018, I hope that human genetics researchers will increase our understanding of the biological mechanisms underlying genome-wide association study data by collaborating with computational and experimental scientists.

\section{Anna Igorevna Podgornaia \\ Merck Research Labs, Boston, MA 02115, USA. \\ Email: anna.podgornaia@merck.com}

When infectious diseases strike, serum from survivors can be administered to others to confer passive immunity. However, serum-based therapy is inefficient and expensive. Moving forward, immunologists and public health officials should strengthen their pipelines to identify such survivors and sequence their antibodies, thereby enabling mass production of the protective antibodies.

\section{Jennifer Chen}

Yale School of Medicine, New Haven, CT 06510, USA. Email: jennifer.s.chen@yale.edu

I hope that computational biologists can take part in more multi-institutional projects that involve both computational and experimental researchers from academia and industry. Such collaboration could help us solve real-life practical problems.

\section{Bipin Singh}

School of Engineering and Technology, BML Munjal University, Gurgaon, Haryana 122413, India. Email: bipin.singh@bmu.edu.in

For kidney disease researchers, interdisciplinary collaboration between scientists focusing on renal diseases, cell reprogramming, 3D printing, and biomechanics is urgently needed this year to push forward the development of artificial organs.

\section{Bo Cao}

Core Research Laboratory, The Second Affiliated Hospital, School of Medicine, Xi'an Jiaotong University, Xi'an Shaanxi, 710004, China. Email: bocao@vip.qq.com

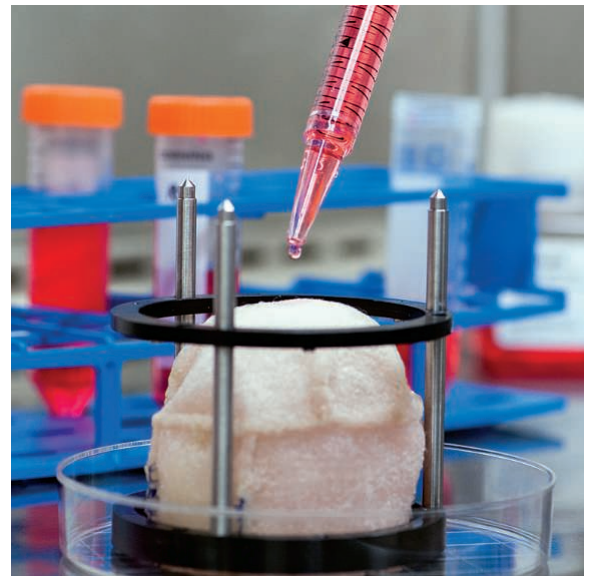

A kidney researcher hopes that collaborations will lead to artificial kidneys similar to this bladder.

Neuromorphic engineers try to create circuits and algorithms that function like the human brain. However, once a basic biological model is implemented, most efforts focus on algorithmic efficiency, which makes the system less realistic. We need more input from neuroscientists. My New Year's resolution is to create a close-knit community of engineers and scientists.

\section{Rishi Raj Singh Sidhu}

Electrical Engineering, Centre of Excellence in IC Design (VIRTUS), Nanyang Technological University, Singapore, 639798, Singapore. Email: rishirajsidhu@gmail.com

\section{Data sharing and analysis}

In an era of next-generation sequencing and artificial intelligence processing of large amounts of biological data, the field of molecular biology should go beyond statistical power and fulfill three criteria: statistical, biological, and clinical significance. Often, statistically significant associations in the laboratory do not have significant clinical utility. We should adopt a clinical perspective that allows clinicians and scientists to harness the power of big data to improve the lives of patients.

Bryce W. Q. Tan

Department of Physiology, National University of Singapore, Singapore 117456, Singapore. Email: brycetan03@hotmail.com

The life sciences community should resolve to generate and interpret data more responsibly. We need to dispel myths and fears surrounding data analytics, adequately train our scientific workforce, and encourage open data and protocol sharing. We need to prevent data dredging, encourage complete and transparent reporting, avoid financial and nonfinancial conflicts, and reward reproducibility as much as novelty.

Prashant Sood

MRC Centre for Medical Mycology, Institute of Medical Sciences, University of Aberdeen, Aberdeen AB25 2ZD, UK. Email: drprashantsood@gmail.com

This year, geologists should emphasize observation-driven over model-driven science. Our models may speak to physically possible hypotheses, but they often fail to be predictive.

\section{Stuart Parker}

Idaho State University, Pocatello, ID 83204, USA. Email: parkstua@isu.edu

My New Year's resolution for psychology and neuroscience is to move beyond a "onesize-fits-all" approach to mental health treatments. We could improve treatment effectiveness by taking individual variability into account. In the next year, I hope that the field works to identify neurological, genetic, and psychological markers of who will best respond to specific interventions or combinations of interventions.

\section{Matthew A. Scult}

Department of Psychology and Neuroscience. Duke University, Durham, NC 27708, USA. Email: matthew.scult@duke.edu

In 2018, I think my field of cancer nanotechnology could benefit from more complete reporting of experimental details, particularly nanoparticle concentrations, animal experiment details, and cell experiment details. Most publications 
provide minimum explanation in these areas, which can make reproducing the findings difficult.

\section{Desiree Van Haute \\ Jefferson, AR 72114, USA. \\ Email: desireevanhaute@gmail.com}

My 2018 resolution would involve a community effort by biologists to integrate data from various sources in databases, which would become available to everyone as fast as possible. Analysis of these data by research labs with diverse interests would lead to an explosion of scientific discoveries.

\section{Nikos Konstantinides}

Department of Biology, New York University, New York, NY 10003, USA. Email: nk1845@nyu.edu

I wish that the field of education research could come together to (finally) agree upon a common core. Without agreement on the foundations of learning and teaching, the field remains divided and of limited use to practitioners and decision-makers.

Beat A. Schwendimann

Berkeley, CA 94720, USA

Email: beat.schwendimann@gmail.com

\section{Affordable research benefits}

Arsenic in rice is a huge problem in Southeast Asia. My New Year's resolution for environmental scientists is to come up with low-cost and easily applicable solutions that allow local farmers to grow safe rice crops.

\section{Sudhakar Srivastava}

Institute of Environment and Sustainable

Development, Banaras Hindu University, Varanasi, Uttar Pradesh 221005, India.

Email: sudhakar.srivastava@gmail.com
At its current cost, there is no hope that next-generation sequencing will be accessible enough to affect medical diagnosis. Sequencing has become a profit-driven business. This year, biotechnology companies should take seriously the task of creating a $\$ 100$ genome, a cost directly controlled by reagent pricing. A lower price tag would make personalized medicine a global possibility, give better data sets for population studies, and make academic research more feasible.

\section{Raffaele Fiorenza}

Sequencing Operations Lab, New York Genome Center, New York, NY 10013, USA.

Email: ralphie.fiorenza@gmail.com

This year, we need to discuss how we will achieve sustainable and affordable oncology with relevant stakeholders, from consumers, researchers, and clinicians to leaders in industry and government. Exploring new funding models and financial structures that continue to incentivize discovery and innovation will be critical for our continued success in treating latestage cancer.

\section{Ken Dutton-Regester}

QIMR Berghofer Medical Research Institute, Brisbane, QLD 4006, Australia.

Email: ken.dutton-regester@qimrberghofer.edu.au

\section{Diversity in science}

My research resolution for ecology would be to ensure that those who have the chance to research biodiversity are as diverse as the communities we study. Ecologists need to do more to recognize the contributions of women and minority researchers and to break down systemic barriers to their

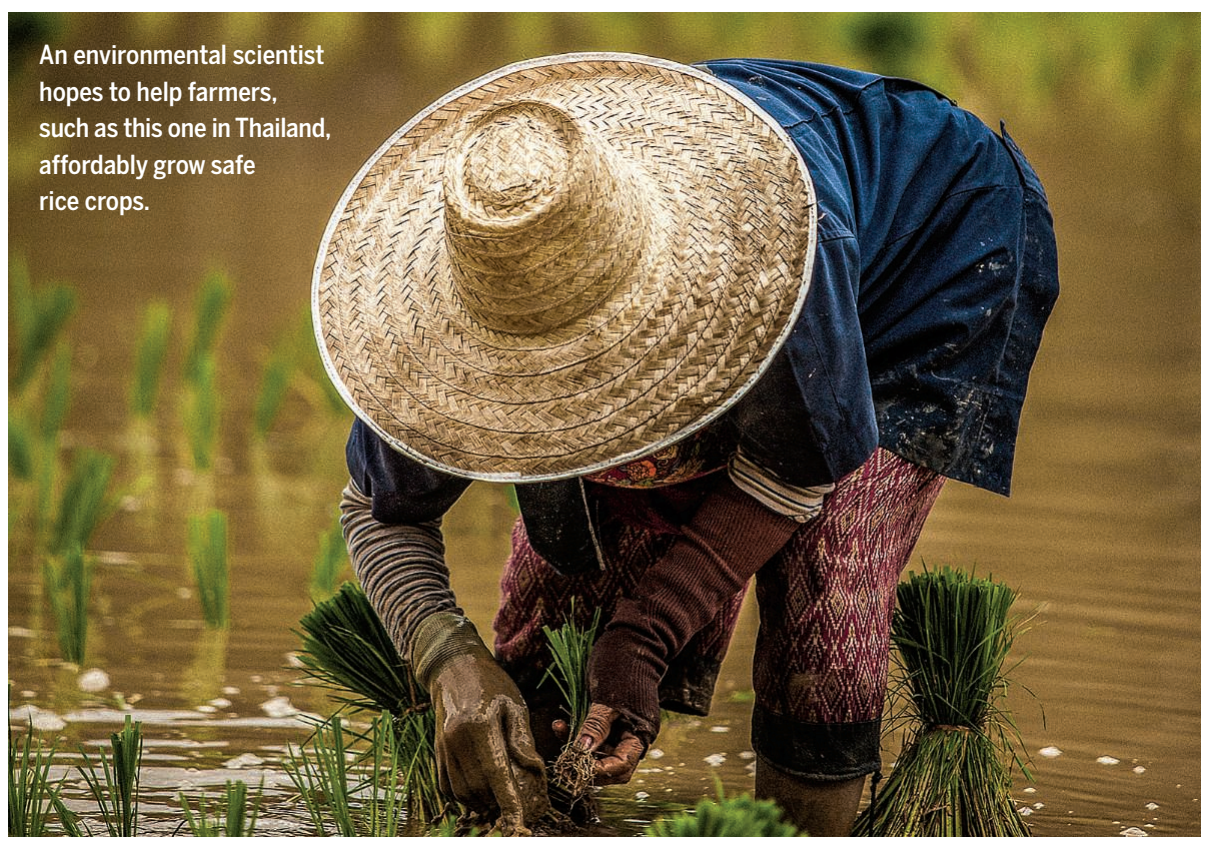

inclusion, such as the prevalence of unpaid "voluntary" field experience positions. We study behavior and species interactions in the field; let's be as aware of our own biases.

\section{Rachel Hale}

Ocean and Earth Science, National Oceanography Centre Southampton, University of Southampton Waterfront Campus, Southampton, Hampshire SO14 3ZH. UK. Email: r.hale@soton.ac.uk

In 2018, instead of focusing on postdoc positions, physics advisers at research institutions should customize career plans for their Ph.D. candidates that take into account each individual's goals, interests, and abilities.

\section{Emre Ozan Polat}

ICFO-The Institute of Photonic Sciences, 08860 Barcelona, Spain.Email: emre-ozan.polat@icfo.es

Postdoctoral fellows are a major driving force of biomedical research, but many postdocs are undercompensated and underrecognized. Our field should remedy this by acknowledging their role as intellectually independent scientists. More invitations could be directly extended to postdocs to pen commentaries, serve as panelists, review grant proposals, and referee manuscripts. Many postdocs already assist their lab heads in review tasks; inviting them to formally serve as reviewers will be a major step toward better postdoc recognition.

\section{Edward Lau}

School of Medicine, Stanford University, Palo Alto, CA 94305, USA. Email: lau1@stanford.edu

My New Year's resolution for ecologists: Pay more attention to the science produced by women and underrepresented minorities. Ensure diversity of keynote speakers, panels, journal editors, and awards. Insist upon field excursions and labs that are supportive and inclusive so that all trainees can thrive.

\section{Audrey L. Mayer}

SFRES, Michigan Technological University, Houghton, MI 49931, USA. Email: almayer@mtu.edu

Although biology graduate programs now have a balance of men and women, the leaky pipeline persists. One challenge to increasing diversity is ongoing sexual harassment on campus, at conferences, and during field work. Such a hostile environment prevents us from creating an inclusive community. Everyone, men in particular, must step up to combat sexual harassment.

\section{Easton R. White}

University of California, Davis, Davis, CA 95616, USA Email: eawhite@ucdavis.edu 


\section{Science}

\section{NextGen VOICES: Research resolutions}

Felicia Olmeta-Schult, Lauren Massa Segal, Sam Tyner, Twila Alexandra Moon, Ryan Dz-Wei Chow, Prosanta Chakrabarty, Martin Pacesa, Anna Igorevna Podgornaia, Jennifer Chen, Bipin Singh, Bo Cao, Rishi Raj Singh Sidhu, Bryce W. Q. Tan, Prashant Sood, Stuart Parker, Matthew A. Scult, Desiree Van Haute, Nikos Konstantinides, Beat A. Schwendimann, Sudhakar Srivastava, Raffaele Fiorenza, Ken Dutton-Regester, Rachel Hale, Emre Ozan Polat, Edward Lau, Audrey L. Mayer and Easton R. White

Science 359 (6371), 26-28.

DOI: $10.1126 /$ science.aar7504

ARTICLE TOOLS

PERMISSIONS http://science.sciencemag.org/content/359/6371/26

http://www.sciencemag.org/help/reprints-and-permissions

Use of this article is subject to the Terms of Service

Science (print ISSN 0036-8075; online ISSN 1095-9203) is published by the American Association for the Advancement of Science, 1200 New York Avenue NW, Washington, DC 20005. The title Science is a registered trademark of AAAS.

Copyright @ 2018 The Authors, some rights reserved; exclusive licensee American Association for the Advancement of Science. No claim to original U.S. Government Works. 